\title{
Boson Fields Under a General Class of Cut-Off Interactions
}

\author{
Raphael HöEgh-KrohN \\ Mathematical Institute, University of Oslo \\ Oslo, Norway
}

Received November 21, 1968

\begin{abstract}
We consider a boson field $\phi(x)$ under an interaction of the form $\int_{|x| \leq r} V\left(\phi_{k}(x)\right) d x$, where $\phi_{k}(x)$ is the momentum cut-off field, and $V(\alpha)$ is a continuous bounded function. Under a weak regularity condition on $V(\alpha)$, we prove that the total energy operator is self adjoint, that the asymptotic fields exist and that the scattering operator exists.
\end{abstract}

\section{Introduction}

The object of this paper is to study a wide class of quantum fields where the interaction is given by a local relativistic interaction with a momentum and space cut-off. The fields will be self interacting boson fields, where the energy operator is given in the form

$$
H=H_{0}+\int_{|x| \leqq r} V\left(\phi_{k}(x)\right) d x .
$$

$H_{0}$ is the free energy operator of a free boson field of strictly positive mass $m . V(\alpha)$ is a real function of a real variable $\alpha$, such that $V(\alpha)$ is the Fourier transform of a finite measure. $\phi_{k}(x)$ is the free field with a momentum cut-off at $k$. In two dimensional space time GuImu [2] has investigated the case where $V$ is a semibounded polynomial, and he was in this case able to remove the momentum cut-off. The case where $V(\alpha)=\lambda \alpha^{4}$ and still in dimension two, can be treated more thoroughly, as shown by JAFFE and GLrmM [3].

One can in this case after removal of the momentum cut-off prove that the total energy operator is self adjoint on the intersection of the domains of its free and interacting part. For the case where $V(\alpha)$ is a semibounded polynomial but in dimension four and with a momentum cut-off JAFFe, LANFond and Wightman [4] were able to prove that the total energy is a self adjoint operator.

We shall prove that in the case $V$ is the Fourier transform of a finite measure, the interaction will be bounded, and so there is no problem with the self adjointness of $H$. But the main object of this paper is to prove existence of asymptotic fields, and the existence of the scattering 
operator. Some of the methods are related to the methods used in Refs. $[5-7]$.

\section{The Total Energy Operator}

We shall use the Fock space representation. The Fock space $\mathscr{F}$ is a Hilbert space where the elements are sequences of functions $f=\left\{f_{0}, f_{1}, \ldots\right\}$ where $f_{n}\left(p_{1}, \ldots, p_{n}\right)$ is a symmetric function of $n$-variables $p_{1}, \ldots, p_{n} ; p_{i} \in R^{3}$. The inner product in $\mathscr{F}$ is given by

$$
(f, g)=\sum_{n=0}^{\infty} n ! \int \cdots \int \bar{f}_{n}\left(p_{1}, \ldots, p_{n}\right) g_{n}\left(p_{1}, \ldots, p_{n}\right) \frac{d p_{1}}{\omega\left(p_{1}\right)} \cdots \frac{d p}{\omega\left(p_{n}\right)}
$$

where $\omega(p)=\left(p^{2}+m^{2}\right)^{1 / 2}$ and $m>0$. The annihilation operator $a(p)$ is defined by

$$
(a(p) f)_{n}\left(p_{1}, \ldots, p_{n}\right)=(n+1) \omega(p)^{-1 / 2} f_{n+1}\left(p, p_{1}, \ldots, p_{n}\right) .
$$

The creation operator $a^{*}(p)$ is the formal adjoint of $\alpha(p)$, and we have

$$
\left[a(p), a^{*}\left(p^{\prime}\right)\right]=\delta\left(p-p^{\prime}\right) .
$$

The free energy operator $H_{0}$ is defined by

$$
\left(H_{0} f\right)_{n}\left(p_{1}, \ldots, p_{n}\right)=\sum_{i=1}^{n} \omega\left(p_{i}\right) f_{n}\left(p_{1}, \ldots, p_{n}\right) .
$$

$H_{0}$ is obviously self adjoint on its natural domain of definition $D_{0}$.

Let $h \in L_{\mathbf{2}}\left(R^{3}\right)$. It is well known that $a(h)=\int a(p) h(p) d p$ and $a^{*}(h)=\int a^{*}(p) h(p) d p$ are closed operators with domains containing $D_{0}$, and $a^{*}(\bar{h})$ is the adjoint of $a(h)$. Moreover, $a(h)+a^{*}(\bar{h})$ is essentially self adjoint on $D_{0}$.

The cut-off field operators are given by

$$
\phi_{k}(x)=2^{-1 / 2}(2 \pi)^{-3 / 2} \int\left(e^{i p x} a(p)+e^{-i p x} a^{*}(p)\right) \omega_{k}(p)^{-1 / 2} d p
$$

where $\omega_{k}(p)^{-1 / 2}=\omega(p)^{-1 / 2}$ for $|p| \leqq k$ and $\omega_{k}(p)^{-1 / 2}=0$ for $|p|>k$. By the remark above $\phi_{k}(x)$ is a self adjoint operator with domain containing $D_{0}$, and it is essentially self adjoint on $D_{0}$.

The interaction $V$ is formally given by

$$
V=\int_{|x| \leqq r} V\left(\phi_{k}(x)\right) d x
$$

We assume that $V$ is the Fourier transform of a finite measure

We now define

$$
V(\alpha)=\int e^{i t \alpha} d \mu(t), \quad \int d|\mu|<\infty \text {. }
$$

$$
V=\int_{|x| \leqq r} d x \int d \mu(t) e^{i t \phi_{k}(x)}
$$

where the integrals are strong integrals, i.e. strong limits of approximating Riemann sums. That the strong integrals above exist follows from the fact that $e^{i t \phi_{k}(x)}$ is an operator which is uniformly bounded and 
strongly continuous in $t$ and $x$. Since $\phi_{k}(x)$ is self adjoint, $e^{i t \phi_{k}(x)}$ is a unitary operator which depends strongly continuously on $t$, and

$$
e^{i t \phi_{k}(x)}=U(-x) e^{i t \phi_{k}(0)} U(x) \text {, }
$$

where $U(x)$ is the strongly continuous unitary group defined by

$$
(U(x) f)_{n}\left(p_{1}, \ldots, p_{n}\right)=e^{i \sum_{j}^{h} x p_{j}} f_{n}\left(p_{1}, \ldots, p_{n}\right) .
$$

Theorem 2.1. Let $\mu$ be a finite measure on the real line. Then

$$
V=\int_{|x| \leqq r} d x \int d \mu(t) e^{i t \phi_{k}(x)}
$$

where the integrals are strong integrals, defines a bounded operator on $\mathscr{F}$. Let

$$
V(\alpha)=\int d \mu(t) e^{i t \alpha} .
$$

If $V(\alpha)$ is real then $V$ is self adjoint, and if $V(\alpha)$ is positive then $V$ is positive.

Proof. We have already seen that the strong integrals defining $V$ exist. That $\mu$ is a finite measure implies that $V$ is a bounded operator. That $V(\alpha)$ is real implies that $\mu(-t)=\bar{\mu}(t)$, which again gives us that $V$ is self adjoint. Let $V(\alpha)$ be a positive function, and let $E_{\lambda}(x)$ be the spectral resolution of $\phi_{k}(x)$. For $f \in \mathscr{F}$ we get

$$
\begin{aligned}
(f, V f) & =\int_{|x| \leqq r} d x \int d \mu(t) \int d\left(f, E_{\lambda}(x) f\right) e^{i t \lambda} \\
& =\int_{|x| \leqq r} d x \int d\left(f, E_{\lambda}(x) f\right) V(\lambda)
\end{aligned}
$$

which is positive since $V(\lambda)$ is positive. This proves the theorem.

\section{The Asymptotic Fields}

We now assume that $V(\alpha)$ is a real function. Since $V$ then is a bounded self adjoint operator we get that

$$
H=H_{0}+V
$$

is a self adjoint operator with the same domain $D_{0}$ as $H_{0}$. Therefore $e^{i t H}$ as well as $e^{i t H_{0}}$ leaves $D_{0}$ invariant. For $h \in L_{2}$ we define

$$
a_{t}^{\#}(h)=e^{-i t H} e^{i t H_{0}} a^{\#}(h) e^{-i t H_{0}} e^{i t H}
$$

where $a^{\#}(h)$ stands for $a^{*}(h)$ or $a(h)$. $a_{t}^{\#}(h)$ is unitarily equivalent to the closed operator $a^{\#}(h)$, hence it is a closed operator. Since $e^{i t H}$ and $e^{-i t H_{0}}$ leaves $D_{0}$ invariant and $D_{0}$ is contained in the domain of $a^{\#}(h)$, we find that $D_{0}$ also is contained in the domain of $a_{t}^{\#}(h)$.

Lemma 3.1. Let $D_{1 / 2}$ be the domain of $H_{0}^{1 / 2}$. Let $h \in L_{2}\left(R^{3}\right)$, then $a_{t}^{\#}(h)$ is a closed operator with domain containing $D_{1 / 2}$. Moreover $a_{t}^{\#}(h)$ maps $D_{1 / 2}$ into $\mathscr{F}$ uniformly bounded in $t$ and $h$ with respect to the natural norm 
in $D_{1 / 2}$. Let $g$ and $h$ be in $L_{2}\left(R^{3}\right)$, then $a_{t}^{\#}(h)$ maps $D_{0}$ into the domain of $a_{t}^{\#}(g)$, and $a_{t}^{\#}(g) a_{t}^{\#}(h)$ maps $D_{0}$ into $\mathscr{F}$ uniformly bounded in $t, g$ and $h$ with respect to the natural norm in $D_{0}$.

Proof. Since $V$ is bounded there exist two constants $a$ and $b$ such that

$$
H_{0}+1 \leqq H+a \leqq H_{0}+b \text {. }
$$

$D_{1 / 2}$ is the completion of $D_{0}$ with respect to the norm $\left(f,\left(H_{0}+1\right) f\right)^{1 / 2}$. Using the inequalities above we see that the norm giving the domain of $H_{0}^{1 / 2}$ is equivalent to the norm giving the domain of $(H+a)^{1 / 2}$. Therefore $D_{1 / 2}$ is also the domain of $(H+a)^{1 / 2}$, so that $e^{i t H}$ as well as $e^{i t H_{0}}$ leaves $D_{1 / 2}$ invariant. It is well known that $D_{1 / 2}$ is contained in the domain of $a^{\#}(h)$, and for $f \in D_{1 / 2},\left\|a^{\#}(h) f\right\| \leqq c\|h\|_{2}\left\|\left(H_{0}+1\right)^{1 / 2} f\right\|$ where $c$ is a constant depending only on $m$. Hence we get that $D_{1 / 2}$ is contained in the domain of $a_{t}^{\#}(h)$ and for $f \in D_{1 / 2}$,

$$
\begin{aligned}
\left\|a_{t}(h) f\right\| & \leqq c\|h\|_{2}\left\|\left(H_{0}+1\right)^{1 / 2} e^{-i t H_{0}} e^{i t H} f\right\| \\
& =c\|h\|_{2}\left\|\left(H_{0}+1\right)^{1 / 2} e^{i t H} f\right\| \\
& \leqq c\|h\|_{2}\left\|(H+a)^{1 / 2} e^{i t H} f\right\| \\
& =c\|h\|_{2}\left\|(H+a)^{1 / 2} f\right\| \\
& \leqq c\|h\|_{2}\left\|\left(H_{0}+b\right)^{1 / 2} f\right\| .
\end{aligned}
$$

This proves the first part of the lemma, to prove the second part we proceed as follows. It is well known that $a^{\#}(h)$ maps $D_{0}$ into the domain of $a^{\#}(g)$ and for $f \in D_{0}$,

$$
\left\|a^{\#}(g) a^{\#}(h) f\right\| \leqq c^{\prime}\|h\|_{2}\|g\|\left\|\left(H_{0}+1\right) f\right\|
$$

where $c^{\prime}$ depends only on $m$.

Since $e^{-i t H_{0}}$ and $e^{i t H}$ leaves $D_{0}$ invariant, we see that $\alpha^{\#}(h) e^{-i t H_{0}} e^{t i H}$ maps $D_{0}$ into the domain of $a^{\#}(g)$. Hence $a_{t}^{\#}(h)$ maps $D_{0}$ into the domain of $a_{t}^{\#}(g)$ and for $f \in D_{0}$

$$
\begin{aligned}
\left\|a_{t}^{\#}(g) a_{t}^{\# \#}(h) f\right\| & \leqq c^{\prime}\|g\|_{2}\|h\|_{2}\left\|\left(H_{0}+1\right) e^{i t H_{0}} e^{i t H} f\right\| \\
& =c^{\prime}\|g\|_{2}\|h\|_{2}\left\|\left(H_{0}+1\right) e^{i t H} f\right\| \\
& \leqq c^{\prime}\|g\|_{2}\|h\|_{2}\left\|\left(H+a^{\prime}\right) e^{i t H} f\right\| \\
& =c^{\prime}\|g\|_{2}\|h\|_{2}\left\|\left(H+a^{\prime}\right) f\right\| \\
& \leqq c^{\prime}\|g\|_{2}\|h\|_{2}\left\|\left(H_{0}+b^{\prime}\right) f\right\| .
\end{aligned}
$$

The last two inequalities comes from the fact that $V$ is bounded. This proves the lemma.

Let $\phi$ and $\psi$ be in $D_{0}$ and $h$ in $L_{2}\left(R^{3}\right)$. Consider the function of one variable $t$ given by:

$$
\left(\phi, a_{t}^{\#}(h) \psi\right)=\left(e^{-i t H_{0}} e^{i t H} \phi, a^{\#}(h) e^{-i t H_{0}} e^{i t H} \psi\right) .
$$


Consider also the function of two variables given by

$$
\begin{aligned}
& \left(e^{-i s H_{0}} e^{i s H} \phi, a^{\#}(h) e^{-i t H_{0}} e^{i t H} \psi\right) \\
= & \left(a^{\#}(h)^{*} e^{-i s H_{0}} e^{i s H} \phi, e^{-i t H_{0}} e^{i t H} \psi\right) .
\end{aligned}
$$

We see that for $s=t$ this is the function given in (3.2). Since $e^{i t H}$ leaves $D_{0}$ invariant, we find that (3.3) is differentiable both with respect to $s$ and $t$. The partial derivatives are given respectively by

and

$$
\left(e^{-i s H_{0}} i V e^{i s H} \phi, a^{\#}(h) e^{-i t H_{0}} e^{i t H} \psi\right)
$$

$$
\left(a^{\#}(h)^{*} e^{-i s H_{0}} e^{i s H} \phi, e^{-i t H_{0}} i V e^{i t H} \psi\right) .
$$

Using that $V$ is a bounded operator and $a^{\#}(h)$ as well as $a^{\#}(h)^{*}$ are closed operators with domains containing $D_{0}$, and that $e^{-i t H_{0}}$ as well as $e^{i t I I}$ leaves $D_{0}$ invariant, we get that both the partial derivatives are continuous in $s$ and $t$. This gives us that (3.2) is a differentiable function of $t$ and

$$
\begin{aligned}
\frac{d}{d t}\left(\phi, a_{t}^{\#}(h) \psi\right)= & \left(e^{-i t H_{0}} i V e^{i t H} \phi, a^{\#}(h) e^{-i t H_{0}} e^{i t H} \psi\right) \\
& +\left(a^{\#}(h)^{*} e^{-i t H_{0}} e^{i t H} \phi, e^{-i t H_{0}} i V e^{i t H} \psi\right) .
\end{aligned}
$$

It is well known that the commutation relations of $H_{0}$ and $a^{\#}(h)$ may be given in the form

$$
e^{i t H_{0}} a^{\#}(h) e^{-i t H_{0}}=a^{\#}\left(h_{ \pm t}\right)
$$

where + goes with $a^{*}$ and - with a and $h_{t}(p)=e^{i t \omega(p)} h(p)$. Using this the formula above may be written.

$$
\begin{aligned}
\frac{d}{d t}\left(\phi, a_{t}^{\#}(h) \psi\right)= & \left(i V e^{i t H} \phi, a^{\#}\left(h_{ \pm t}\right) e^{i t H} \psi\right) \\
& +\left(a^{\#}\left(h_{ \pm t}\right)^{*} e^{i t H} \phi, i V e^{i t H} \psi\right) .
\end{aligned}
$$

Lemma 3.6. Let $|\mu|$ be the absolute value of $\mu$. If $|\mu|$ has a first order moment, then for $h \in L_{2}\left(R^{3}\right), V$ leaves the domain of $a^{\#}(h)$ invariant and

$$
\left\|\left[a^{\#}(h), V\right]\right\| \leqq C \cdot \sup _{x}\left|\int h(p) \omega_{k}(p)^{-1 / 2} e^{i x p} d p\right| .
$$

The constant $C$ depends only on the first order moment of $|\mu|$ and on $r$. Proof. From the commutation relations of $a^{\# \#}(h)$ we get

$$
\begin{aligned}
{\left[a(h), e^{i s \phi_{k}(x)}\right] } & =i s\left[a(h), \phi_{k}(x)\right] e^{i s \phi_{k}(x)} \\
& =2^{-1 / 2}(2 \pi)^{-2 / 3} i s \int h(p) \omega_{k}(p)^{-1 / 2} e^{-i p x} d p \cdot e^{i s \phi_{k}(x)} .
\end{aligned}
$$

So that $\left[a(h), e^{i s \phi_{k}(x)}\right]$ is a bounded operator, and therefore $e^{i s \phi_{k}(x)}$ leaves invariant the domain of $a(h)$. Since $e^{i t \phi_{k}(x)}$ is strongly continuous, $V \psi$ may be approximated strongly by Riemann sums of the form 
$\sum_{n m} C_{n m} e^{i s_{n} \phi_{k}\left(x_{m}\right)} \psi$. Let $\psi$ be in the domain of $a(h)$, then

$$
\begin{aligned}
& a(h) \sum_{n m} C_{n m} e^{i s_{n} \phi_{k}\left(x_{m}\right)} \psi=\sum_{n m} C_{n m} e^{i s_{n} \phi_{k}\left(x_{m}\right)} a(h) \psi \\
& \quad+\sum_{n m} C_{n m} 2^{-1 / 2}(2 \pi)^{-3 / 2} i s_{n} \int h(p) \omega_{k}(p)^{-1 / 2} e^{-i x_{m} p} d p e^{i s_{n} \phi_{k}\left(x_{m}\right)} \psi .
\end{aligned}
$$

Since $|\mu|$ has a first order moment, we see the right hand side converges strongly to

$$
\begin{aligned}
V a(h) \psi & +2^{-1 / 2}(2 \pi)^{-3 / 2} \int_{|x| \leqq r} d x \int d \mu(s) i s \\
& \cdot \int h(p) \omega_{k}(p)^{-1 / 2} e^{-i x p} d p e^{i s \phi_{k}(x)} \psi .
\end{aligned}
$$

Since $a(h)$ is a closed operator we get that the left hand side converges strongly to $a(h) V \psi$, and that $V \psi$ is in the domain of $a(h)$. By a direct estimate of the norm of the second term above, we see that the lemma is proved for $a(h)$. The proof for $a^{*}(h)$ is the same up to trivial modifications.

We use the lemma to write (3.5) in the form

$$
\frac{d}{d t}\left(\phi, a_{t}^{\#}(h) \psi\right)=\left(\phi, e^{-i t H}\left[a^{\#}\left(h_{ \pm t}\right), i V\right] e^{i t H} \psi\right) .
$$

Using that $a^{\#}(h)$ is a closed operator and the fact that $V$ leaves the domain of $a^{\#}(h)$ invariant, we see that $e^{-i t H}\left[a^{\#}\left(h_{ \pm t}\right), i V\right] e^{i t I I} \psi$ is strongly continuous for $\psi \in D_{0}$. Integrating both sides of the identity above we thus get

$$
\left(\phi, a_{t}^{\#}(h) \psi\right)-\left(\phi, a^{\#}(h) \psi\right)=\left(\phi, \int_{0}^{t} d s e^{-i s H}\left[a^{\#}\left(h_{ \pm s}\right), i V\right] e^{i s H} \psi\right) \text {. }
$$

Using the estimate for the norm in Lemma 3.6, we find that the integral above gives a bounded operator. This gives the formula

$$
a_{t}^{\#}(h)-a^{\#}(h)=\int_{0}^{t} d s e^{-i s H}\left[a^{\#}\left(h_{ \pm s}\right), i V\right] e^{i s H}
$$

where the integral is taken in the strong sense.

Theorem 3.8. Let $h \in L_{\mathbf{2}}\left(R^{3}\right)$ and $\phi \in D_{1 / \mathbf{2}}$. Then $a_{t}^{\#}(h) \phi$ converges strongly as $t$ tends to $\pm \infty$. The limit operators $a_{ \pm}^{\#}(h)$ are closable operators defined on $D_{1 / 2}$, and $a_{ \pm}(h)$ maps $D_{1 / 2}$ into $\mathscr{F}$ uniformly bounded in $h$ with respect to the natural norm in $D_{1 / 2}$. If we denote the closure of $a_{ \pm}^{\#}(h)$ also by $a_{ \pm}^{\#}(h)$, then $a_{ \pm}^{*}(\bar{h})$ and $a_{ \pm}(h)$ is the adjoint of each other.

Proof. Let $L_{0}$ be the dense subspace in $L_{2}$, consisting of functions $h$, such that $h=0$ in a neighbourhood of $p=0$, and $h(p) \omega_{k}(p)^{-1 / 2}$ is in $C_{0}^{\infty}$. It follows from the estimate in Lemma 3.6 that for $h \in L_{0}$ $\left\|\left[a^{\#}\left(h_{ \pm \delta}\right), i V\right]\right\|$ tends to zero faster than any inverse power of $s$. By (3.7) $a_{t}^{\#}(h)-a^{\#}(h)$ converges in norm as $t$ tends to $\pm \infty$. Since $\phi \in D_{1 / 2}$, hence in the domain of $a^{\#}(h)$, this gives that $a_{t}^{\#}(h) \phi$ converges strongly. 
By Lemma $3.1 a_{t}^{\#}(h) \phi$ is uniformly bounded in $t$ and $h$ hence we get that $a_{t}^{\#}(h) \phi$ converges strongly for all $h \in L_{2}$. By the same lemma $a_{t}^{\#}(h)$ is uniformly bounded in $t$ and $h$ as a mapping from $D_{1 / 2}$ into $\mathscr{F}$. Hence the limits $a_{ \pm}^{\#}(h)$ must also map $D_{1 / 2}$ into $\mathscr{F}$ uniformly bounded in $h$ with respect to the natural norm in $D_{1 / 2}$. Let $\phi$ and $\psi$ be in $D_{1 / 2}$. Since

$$
\left(a_{t}^{*}(\bar{h}) \phi, \psi\right)=\left(\phi, a_{t}(h) \psi\right)
$$

we get by strong convergence that

$$
\left(a_{ \pm}^{*}(\bar{h}) \phi, \psi\right)=\left(\phi, a_{ \pm}(h) \psi\right) .
$$

Hence the adjoint of $a_{ \pm}^{\#}(h)$ is densely defined, and therefore $a_{ \pm}^{\#}(h)$ is closable. It follows also from this identity that $a_{ \pm}^{*}(h)$ and $a_{ \pm}(h)$ are the adjoints of each other.

From now on $a_{ \pm}^{\#}(h)$ denotes the closed operators.

Theorem 3.9. Let $g$ and $h$ be in $L_{2}\left(R^{3}\right)$. Then a $a_{1}(h)$ maps $D_{0}$ into the domain of $a_{ \pm}^{\#}(g)$, and $a_{ \pm}^{\#}(g) a_{ \pm}^{\#}(h)$ maps $D_{0}$ into $\mathscr{F}$ uniformly bounded in $g$ and $h$ with respect to the natural norm on $D_{0}$. $a_{ \pm}^{\#}(h)$ satisfies the same commutation relations on $D_{0}$ as do $a^{\#}(h)$ on $D_{0}$.

$H$ and $a_{ \pm}^{\#}(h)$ satisfy the same commutation relations as do $H_{0}$ and $a^{\#}(h)$ in the sense that

on $D_{1 / 2}$.

$$
\begin{aligned}
& e^{i t H} a_{ \pm}(h) e^{-i t H}=a_{ \pm}\left(h_{-t}\right) \\
& e^{i t H} a_{ \pm}^{*}(h) e^{-i t H}=a_{ \pm}^{*}\left(h_{t}\right)
\end{aligned}
$$

Proof. Let $\phi$ and $\psi$ be in $D_{0}$. By Theorem $3.8\left(\phi, a_{t}^{\#}(g) a_{t}^{\#}(h) \psi\right)$ converges to $\left(a_{ \pm}^{\#}(g)^{*} \phi, a_{ \pm}^{\#}(h) \psi\right)$. By Lemma 3.1

hence

$$
\left|\left(\phi, a_{t}^{\#}(g) a_{t}^{\#}(h) \psi\right)\right| \leqq C\|g\|_{2}\|h\|_{2}\|\phi\|\left\|\left(H_{0}+1\right) \psi\right\|,
$$

$$
\left|\left(a_{ \pm}^{\#}(g)^{*} \phi, a_{ \pm}^{\#}(h) \psi\right)\right| \leqq C\|g\|_{2}\|h\|_{2}\|\phi\|\left\|\left(H_{0}+1\right) \psi\right\| .
$$

From this we get that $a_{ \pm}^{\#}(h) \psi$ is in the domain of $a_{ \pm}^{\#}(g)$, and the uniform boundedness with respect to $g$ and $h$. Moreover we see that $\left(\phi, a_{t}^{\#}(g) a_{t}^{\#}(h) \psi\right)$ converges to $\left(\phi, a_{ \pm}^{\#}(g) a_{ \pm}^{\#}(h) \psi\right)$. This gives us that $\left(\phi,\left[a_{t}^{\#}(g), a_{t}^{\#}(h)\right] \psi\right)$ converges to $\left(\phi,\left[a_{ \pm}^{\#}(g), a_{ \pm}^{\#}(h)\right] \psi\right)$. The fact that $\left[a_{t}^{\#}(g), a_{t}^{\# \#}(h)\right]$ is bounded and independent of $t$ proves the first part of the theorem.

To prove the second part of the theorem we observe that

$$
e^{i t I I} a_{s}^{\#}(h) e^{-i t H}=a_{s-t}^{\#}\left(h_{ \pm t}\right)
$$

on $D_{1 / 2}$. Since $e^{-i t H}$ leaves $D_{1 / 2}$ invariant, we may take the strong limits on $D_{1 / 2}$ of the identity above, and this proves the rest of the theorem.

Theorem 3.10. Let $\phi$ be an eigenvector of $H$. Then for any $h$ in $L_{2}$

$$
a_{ \pm}(h) \phi=0 \text {. }
$$


Proof. Let $\lambda$ be the corresponding eigenvalue. Then

$$
a_{t}(h) \phi=e^{-i t(H-\lambda)} a\left(h_{-t}\right) \phi .
$$

Since $\phi$ is an eigenvector of $H$ it is in $D_{1 / 2}$. Hence it is enough to prove that $a\left(h_{-t}\right)$ tends strongly to zero on $D_{1 / 2}$ as $t$ tends to $\pm \infty$. By the estimate

$$
\|a(h) \psi\| \leqq C\|h\|_{2}\left\|\left(H_{0}+1\right)^{1 / 2} \psi\right\|
$$

it is enough to prove that $a\left(h_{-t}\right) \psi$ tends strongly to zero when $h$ is in a dense set in $L_{2}$ and $\psi$ is in a dense set in $D_{1 / 2}$. So let $h \in C_{0}^{\infty}$ and $h(p)=0$ in a neighbourhood of $p=0$, and let $\psi=\left\{\psi_{0}, \psi_{1}, \ldots \psi_{n}, 0,0 \ldots\right\}$ where $\psi_{n} \in C_{0}^{\infty}$. From the definition of $a(h)$ we find that $\left\|a\left(h_{-t}\right) \psi\right\|$ tends to zero faster than any inverse power of $t$. This proves the theorem.

\section{The Asymptotic Decomposition of $\boldsymbol{I}$ and the Scattering Operator}

Let $V_{ \pm}^{0}$ be the set of vectors in $\mathscr{F}$ which is annihilated by $a_{ \pm}(h)$ for all $h \in L_{2}\left(R^{3}\right)$. Since $a_{ \pm}(h)$ are closed operators $V_{ \pm}^{0}$ is a closed linear subspace of $\mathscr{F}$. From the commutation relations of $a_{ \pm}(h)$ and $H$, as given in Theorem 3.9, it follows that $V_{ \pm}^{0}$ is an invariant subspace for $H$. Hence $D_{0} \cap V_{ \pm}^{0}$ is dense in $V_{ \pm}^{0}$. From the commutation relations of $a_{ \pm}^{\#}(h)$, as given in Theorem 3.9, we see that $a_{ \pm}^{*}(h)$ are bounded operators on $D_{0} \cap V_{ \pm}^{0}$ for all $h \in L_{2}\left(R^{3}\right)$. Since $a_{ \pm}^{*}(h)$ are closed operators for $h \in L_{2}$ we get that $V_{ \pm}^{0}$ is in the domain of $a_{ \pm}^{*}(h)$ for $h \in L_{2}$. Let $V_{ \pm}^{1}$ be the smallest closed subspace of $\mathscr{F}$ containing $a_{ \pm}^{*}(h) V_{ \pm}^{0}$ for all $h \in L_{2}$. It follows from the commutation relations of $a_{ \pm}^{*}(h)$ and $H$ that $V_{ \pm}^{1}$ reduces $H$, and hence $D_{0} \cap V_{ \pm}^{1}$ is dense in $V_{ \pm}^{1}$. Using the commutation relations for $a_{ \pm}^{\#}(h)$ we get that $a_{ \pm}^{*}(h)$ as well as $a_{ \pm}(h)$ are bounded operators on $D_{0} \cap V_{ \pm}^{1}$, hence $V_{ \pm}^{1}$ is in the domain of $a_{ \pm}^{*}(h)$ as well as $a_{ \pm}(h)$ for all $h \in L_{2}$. It follows also from the commutation relations of $a_{ \pm}^{\#}(h)$ that $V_{ \pm}^{0} \cap V_{ \pm}^{1}=0$. We now define $V_{ \pm}^{n}$ as the smallest closed subspace containing $a_{ \pm}^{*}(h) V_{ \pm}^{n-1}$ for all $h \in L_{2}$. It follows as above that $V_{ \pm}^{n}$ reduces $H$ and is contained in the domain of $a_{ \pm}^{\#}(h)$. It follows also from the commutation relations of $a_{ \pm}^{\#}(h)$ that for all $h \in L_{2}, a_{ \pm}(h)$ maps $V_{ \pm}^{n}$ into $V_{ \pm}^{n-1}$, and that $\sum_{k=0}^{n} V_{ \pm}^{k}$ is the largest closed subspace annihilated by all operators of the form $a_{ \pm}\left(h_{1}\right) \ldots a_{ \pm}\left(h_{n+1}\right)$ for $h_{1}, \ldots, h_{n+1}$ in $L_{2}$.

\section{Lemma 4.1.}

$$
\mathscr{F}=\sum_{k=0}^{\infty} V_{ \pm}^{k}
$$

where the sum is a direct sum of mutually orthogonal subspaces.

Proof. Let $\phi \in V_{ \pm}^{n}$ and $\psi \in V_{ \pm}^{m}$ and assume that $n>m$. Assume that $\phi$ is of the form $a_{ \pm}^{*}\left(h_{1}\right) \ldots a_{ \pm}^{*}\left(h_{n}\right) \phi_{0}$, where $\phi_{0} \in V_{ \pm}^{0}$.

$$
\left(a_{ \pm}^{*}\left(h_{1}\right) \ldots a_{ \pm}^{*}\left(h_{n}\right) \phi_{0}, \psi\right)=\left(\phi_{0}, a_{ \pm}\left(h_{n}\right) \ldots a_{ \pm}\left(h_{1}\right) \psi\right)
$$

which is zero since $n>m$. Hence $V_{ \pm}^{n}$ and $V_{ \pm}^{m}$ are mutually orthogonal. 16 Commun. math. Phys., Vol. 12 
Let $E_{\lambda}$ be the resolution of the identity corresponding to $H$, and let $\omega_{0}$ be the bound on the spectrum of $H$. $\omega_{0}$ is finite since $V$ is bounded. Since $D_{0}$ is contained in the domain of $a_{ \pm}(h), E_{\lambda} \mathscr{F}$ is contained in the domain of $a_{ \pm}(h)$. It follows now from the commutation relations of $a_{ \pm}(h)$ and $H$ that $a_{ \pm}(h) E_{\lambda} \mathscr{F} \subset E_{\lambda-m} \mathscr{F}$ where $m$ is the mass of the free field. For details on this see Lemma 4 and Lemma 5, Section 3 of Ref. [5].

Hence $E_{\lambda} \mathscr{F}$ is contained in $\sum_{k=0}^{n} V_{ \pm}^{k}$ for $\lambda-\omega_{0}<n \cdot m$, where $m$ is the mass of the free field. This proves that $\sum_{k=0}^{\infty} V_{ \pm}^{k}$ is dense in $\mathscr{F}$, and since the sum is a direct sum it must be equal to $\mathscr{F}$. This proves the lemma.

Theorem 4.2. $\mathscr{F}$ decomposes as a tensor product $\mathscr{F}_{ \pm} \otimes V_{ \pm}^{0}$ relative to the asymptotic operators $a_{ \pm}^{\#}(h) . V_{ \pm}^{0}$ may be identified with the subspace of $\mathscr{F}$ annihilated by all operators $a_{ \pm}(h), h \in L_{2}$, and $\mathscr{F}_{ \pm}$is the Fock space constructed with $a_{ \pm}^{\#}(h)$ as creation and annihilation operators. Relative to this decomposition $H$ decomposes as

$$
H=H_{0}^{ \pm} \otimes 1+1 \otimes H_{ \pm}^{0}
$$

where $H_{0}^{ \pm}$is the free energy operator in $\mathscr{F}_{ \pm}$, and $H_{0}^{ \pm}$is the restriction of $H$ to $V_{0}^{ \pm}$.

Proof. Let $\Omega_{ \pm}$be the vacuum state in $\mathscr{F}_{ \pm}$. We then identify $\mathscr{F}$ with $\mathscr{F}_{ \pm} \otimes V_{ \pm}^{0}$ in the following way. Let $\phi \in V_{ \pm}^{n}$ be of the form $\phi=a_{ \pm}^{*}\left(h_{1}\right) \ldots a_{ \pm}^{*}\left(h_{n}\right) \phi_{0}$, where $\phi_{0} \in V_{ \pm}^{0}$, then $\phi$ is mapped into $a_{ \pm}^{*}\left(h_{1}\right) \ldots a_{ \pm}^{*}\left(h_{n}\right) \Omega_{ \pm} \otimes \phi_{0}$. It follows from the commutation relations that this mapping preserves the inner product, hence it is also one to one. Its range is obviously dense in $\mathscr{F}_{ \pm} \otimes V_{ \pm}^{0}$, and it follows from Lemma 4.1 that it is defined on a dense set in $\mathscr{F}$. Hence it extends uniquely to an identification of $\mathscr{F}$ with $\mathscr{F}_{ \pm} \otimes V_{ \pm}^{0}$. This proves the first part of the theorem. The second part of the theorem follows from the commutation relations of $a_{ \pm}^{\#}(h)$ and $H$ as given in Theorem 3.9.

Let us now assume that $H$ has an eigenvector $\Omega$ with the eigenvalue $\omega$. Since $H_{0}$ has a simple eigenvalue which is separated from the rest of the spectrum by an interval of length $m, H$ will have an eigenvector $\Omega$ with eigenvalue $\omega$ if $\|V\|<C$ where $C$ depends only on $m$, such that $\Omega$ as well as $\omega$ depends analytically on $V$ (see for instance Ref. [1]). In our case $\|V\|$ will be small if either the total mass of $|\mu|$ is small or $r$ is small.

If $H$ has an eigenvalue $\Omega$, Theorem 3.10 tells us that $\Omega \in V_{ \pm}^{0}$. In this case it follows from the proof of Theorem 4.2 that we may also identify $\mathscr{F}_{ \pm}$with subspaces of $\mathscr{F}$ by identifying $\Omega_{ \pm}$with $\Omega$. The only difference will be that in the decomposition of $H$, where we get

$$
H=\left(H_{0}^{ \pm}+\omega\right) \otimes 1+1 \otimes H_{ \pm}^{0},
$$


where $H_{0}^{ \pm}$is the free energy operator in $\mathscr{F}_{ \pm}, H_{0}^{ \pm}+\omega$ is the restriction of $H$ to $\mathscr{F}_{ \pm}$, and $H_{ \pm}^{0}$ is still the restriction of $H$ to $V_{ \pm}^{0}$.

At least in the case where $\|V\|<C$, and $\Omega$ depends analytically on $V$, or in the case that $\Omega$ is the only eigenvector of $H$, it is natural to identify $\Omega$ with the physical vacuum. This identification leads to the identification of $a_{ \pm}^{*}\left(h_{1}\right) \ldots a_{ \pm}^{*}\left(h_{n}\right) \Omega$ with the outgoing (incoming) $n$-particle state with momentum distribution given by $h_{1}, \ldots, h_{n}$, and $\mathscr{F}_{ \pm}$with the subspaces corresponding to outgoing (incoming) states. The scattering operator $S$ may now be defined as partial isometry mapping $\mathscr{F}_{\text {_ into } \mathscr{F}} \mathscr{F}_{\text {by }}$

$$
S: a_{-}^{*}\left(h_{1}\right) \ldots a_{-}^{*}\left(h_{n}\right) \Omega \rightarrow a_{+}^{*}\left(h_{1}\right) \ldots a_{+}^{*}\left(h_{n}\right) \Omega
$$

which corresponds to the usual definition of the scattering operator in the $H$-picture in quantum mechanics (see for instance Ref. [1]). The probability amplitude for finding an outgoing $n$-particle state with momentum distribution given by $h_{1}, \ldots, h_{n}$, when we send in $m$-particles with momentum distribution given by $\tilde{h}_{1}, \ldots, \tilde{h}_{m}$ is

$$
\begin{aligned}
& S\left(h_{1}, \ldots, h_{n} \mid \tilde{h}_{1}, \ldots, \tilde{h}_{m}\right) \\
& \quad=\left(a_{+}^{*}\left(h_{1}\right) \ldots a_{+}^{*}\left(h_{n}\right) \Omega, a_{-}^{*}\left(\tilde{h}_{1}\right) \ldots a_{-}^{*}\left(\tilde{h}_{m}\right) \Omega\right) .
\end{aligned}
$$

Unitarity of the $S$-matrix corresponds to whether $\mathscr{F}_{+}=\mathscr{F}_{-}$or not.

\title{
References
}

1. Friedrichs, K. O.: Perturbation of spectra in Hilbert space. Providence: Am. Math. Soc. 1965.

2. Guinu, J.: Boson fields with nonlinear selfinteraction in two dimensions. Commun. Math. Phys. 8, 12-25 (1968).

3. Glinm, J., and A. JAFFe: A $: \lambda \phi^{4}$ : quantum field theory without cut-offs. To appear.

4. JAFFe, A., O. Lanford, and A. S. Wightman: A general class of cut-off model fields. Preprint.

5. HöЕGH-KRoHN, R.: Asymptotic limits in some models of quantum field theory I. J. Math. Phys. Jan. (1969).

6. - Asymptotic limits in some models of quantum field theory II. J. Math. Phys. April (1969).

7. - Asymptotic limits in some models of quantum field theory III. J. Math. Phys. To appear.

\author{
Raphael Höegh-KroHN \\ Mathematical Institute \\ University of Oslo \\ Blindern, Oslo 3, Norwegen
}

